\title{
Diagnostic checking of time series models
}

\author{
P. Sekar \\ Department of Mathematics, Pachaiyappa's College for Men, Kancheepuram-631 501, TN, India \\ cicesekar@yahoo.co.in; icesekar@rediffmail.com
}

\begin{abstract}
Diagnostic checks have become a standard tool for identification of models before forecasting the data. The overall test for lack of fit for autoregressive moving average models proposed by Box and Pierce (1970) and a measure of lack of fit in time series models proposed by Ljung and Box (1978) are considered. In this paper, a modification is made and it is shown that a substantially improved approximation results from a simple improvement of this test. Cumulative periodogram check is also given.
\end{abstract}

Keywords: Time series, ARMA, ARIMA, forecasting.

\section{General}

Extensive literature is available on diagnostic checks for ARMA models (Jenkins \& Watts, 1969; Hokstad, 1983; Box et al., 1994). McLeod and Li (1983) proposed the diagnostic checks using residual autocorrelation. McLeod (1994) obtained new results on the distribution of residual autocorrelation and derived suitable diagnostic checks and illustrated them with an application of Fraser river time series. Ljung (1986) examined the properties of Portmanteau statistic for testing the adequacy of the model for various choices of $m$ where $m$ is the number of autocorrelations. Some of the commonly applied diagnostic checks are discussed subsequently.

To justify the modified diagnostic checking introduced the monthly rainfall data of Chennai city from the month of January 1984 to December 1994 is analyzed.

\section{Overfitting}

One class of diagnostic checks is devised to test adequacy by over fitting. Over fitting involves fitting a more elaborate model, than the one estimated to see, if the inclusion of one or more parameters greatly improves the fit. Extra parameters should be estimated for the more complex model, only where it is feared that the simpler model may require more parameters. However, the method of overfitting by extending the model in a particular direction assumes that model discrepancies are known in advance, which may not be possible in most of the cases. Procedures, less dependent upon such knowledge are based on the analysis of the residuals.

\section{The graph of the residuals}

If the fitted model is appropriate, then the graph of $\left\{\widehat{a}_{t}\right.$, $t=1,2 \ldots n\}$ should resemble that of a white noise process. While it is difficult to identify the correlation structure of $\left\{\hat{a}_{\mathrm{t}}\right\}$ from its graph, deviation of the mean from zero is sometimes clearly indicated by a trend or cyclic component and inconsistency of the variance by fluctuations in $\left\{\widehat{a}_{\mathrm{t}}\right\}$ whose magnitude depends strongly on t.

The next step is to check that the sample autocorrelation function of $\widehat{a}_{1}, \widehat{a}_{2}, \ldots \widehat{a}_{\mathrm{n}}$ behaves as it should, under the assumption that the fitted model is appropriate.

\section{Autocorrelation check}

Brockwell and Davis (1991) have proved that the sample autocorrelations of an identically and independently distributed (i.i.d) sequence $\left\{z_{t}, z_{2} \ldots z_{n}\right\}$ with $E\left(z_{t}^{2}\right)<\infty$ for large $n$ are approximately i.i.d with distribution $N(0,1 / \sqrt{ } n)$. Assuming, therefore, that an appropriate ARMA model is generated by a white noise sequence, the same approximation should be valid for the sample autocorrelation function (ACF) of $\left\{\widehat{a}_{\mathrm{t}} / \mathrm{t}=1,2 \ldots \mathrm{N}\right\}$ defined by

$$
r_{k}(\widehat{a})=\frac{\sum_{t=1}^{n-k}\left(\hat{a}_{t}-\bar{a}\right)\left(\hat{a}_{t+k}-\bar{a}\right)}{\sum_{t=1}^{n}\left(\hat{a}_{t}-\bar{a}\right)^{2}}
$$

where $\mathrm{k}=1,2 \ldots$ and $\bar{a}=n^{-1} \sum_{r=1}^{n-k} \widehat{a}_{t}$

However, because each $\hat{a}_{t}$ is a function of the maximum likelihood estimator $\left(\hat{\varphi}, \hat{\theta}, \widehat{a}_{1}, \widehat{a}_{2}, \ldots . \widehat{a}_{n}\right)$ is not an i.i.d sequence and the distribution of $r_{k}(\widehat{a})$ is not quite the same as in the i.i.d case. In certain cases, recognizable patterns in the estimated ACF of $\hat{a}_{t}$ could point to appropriate modifications in the model. Supposing the form of the model were correct and the true values of AR and MA parameters, namely, $\varphi, \theta$ are known exactly, then by Bartlett's approximation (Bartlett, 1946) to the standard error of ACF, and as illustrated by 
Anderson (1941), the estimated autocorrelations $r_{k}(\widehat{a})$ of the $\widehat{a}_{t}$ 's would be uncorrelated and distributed normally about mean zero and variance $1 / \mathrm{n}$ and hence with a standard error of $\mathrm{n}^{-1 / 2}$. Using these results, the significance of the apparent departure of these autocorrelations from zero may be analyzed. In practical applications to real time data, the true values of the parameters are not known and only the estimated values are available for analysis. The autocorrelations $r_{k}(\hat{a})$ and $\hat{a}_{t}$ 's may yield valuable evidence concerning lack of fit and the nature of model inadequacy.

However, Durbin (1960) pointed out that it might be dangerous to assess the statistical significance of apparent discrepancies of these autocorrelations $r_{k}(\hat{a})$ from their theoretical zero values on the basis of a standard error of $\mathrm{n}^{-1 / 2}$, appropriate to the $r_{k}(\hat{a})$ 's. Subsequently Box and Pierce (1970) have shown that, while in all cases, a reduction in variance can occur for low lags and that at these low lags, the autocorrelations $r_{k}(\widehat{a})$ can be highly correlated and these effects usually disappear rather quickly at higher lags. Thus, the use of $\mathrm{n}^{-1 / 2}$ as the standard error for $\mathrm{r}_{\mathrm{k}}(\widehat{a})$ would underestimate the statistical significance of apparent departures from zero of the autocorrelation at low lags, but could usually be employed for moderate or higher lags. Hence, except at moderate lags, $\mathrm{n}^{-1 / 2}$ must be regarded as supplying an upper level for the standard errors of the $r_{k}(\widehat{a})$ 's rather than the standard errors themselves.

Ljung and Box (1978) made a simple modification of the test of Box and Pierce (1970) which substantially improved approximation that should be adequate for most practical purposes. Godfrey (1979) examined both the above tests and proposed a new approach and some Monte Carlo results on the finite sample size for testing the adequacy of a time series model.

\section{Cumulative periodogram}

In some applications, particularly in the fitting of seasonal time series, it may be feared that the model has not adequately taken into account, the periodic characteristics of the available data. Therefore, one must be on the lookout for the periodic behaviour of the residuals. In such cases, the ACF will not be a sensitive indicator of such departures from randomness because periodic effects will typically dilute themselves among several autocorrelations. The periodogram, on the other hand, is specifically designed for the periodic patterns in a background of white noise. The periodogram of a time series $\left\{a_{t}, t=1,2, \ldots n\right\}$ is defined as

$$
\begin{aligned}
& \mathrm{I}\left(\mathrm{f}_{\mathrm{i}}\right)=\frac{2}{n}\left[\left(\sum_{t=1}^{n} a_{t} \cos 2 \pi f_{i} t\right)^{2}+\left(\sum_{t=1}^{n} a_{t} \sin 2 \pi f_{i} t\right)^{2}\right] \mathrm{i}=1,2 . . \\
& \text { where } \mathrm{f}_{\mathrm{i}}=\frac{i}{n} \text { is the frequency. }
\end{aligned}
$$

Thus, it is a device for correlating the $a_{t}$ with sine and cosine waves of different frequencies. A pattern with frequency $f_{i}$ in the residual is reinforced when correlated with a sine and cosine wave at that same frequency and so produces a large value of $\mathrm{I}\left(\mathrm{f}_{\mathrm{i}}\right)$. It has been shown by Bartlett (1946) that the cumulative periodogram provides an effective means for the detection of non-randomness. Jenkins and Watts (1968) have also discussed in detail the applications of the cumulative periodogram.

The power spectrum $P$ (f) for white noise has a constant value $2 \sigma_{a}{ }^{2}$ over the frequency $0-0.5$ cycles. Consequently, the cumulative spectrum for white noise, namely

$$
P(f)=\int_{0}^{f} p(a) d a
$$

plotted against $f$ is a straight line running from $(0,0)$ to $\left(0.5, \sigma_{a}{ }^{2}\right)$, that is $P(f) / \sigma_{a}{ }^{2}$ is a straight line running from $(0,0)$ to $(0.5,1)$. I(f) produces an estimate of the power spectrum at frequency $f$. In fact, for white noise $E[\mathrm{l}(\mathrm{f})]=$ $2 \sigma_{a}{ }^{2}$ and hence the estimate is unbiased. It follows that

$\frac{1}{n} \sum_{i=1}^{j} I\left(f_{i}\right)$ provides an unbiased estimate of the integrated spectrum $P\left(f_{j}\right)$ and

$$
\mathrm{C}\left(\mathrm{f}_{\mathrm{i}}\right)=\frac{\sum_{i=1}^{j} I\left(f_{i}\right)}{n \hat{\sigma}_{a}^{2}}
$$

is an estimate of $\mathrm{P}\left(\mathrm{f}_{\mathrm{i}}\right) / \widehat{\sigma}_{a}^{2}$ where $\widehat{\sigma}_{a}^{2}$ is an estimate of $\sigma_{\mathrm{a}}{ }^{2}$. $\mathrm{C}\left(\mathrm{f}_{\mathrm{j}}\right)$ is called the cumulative periodogram.

Now if the models were adequate and the parameters known exactly, the $a_{t}$ 's could be computed from the data and would yield a white noise series. For a white noise series, the plot of $C\left(f_{j}\right)$ against $f_{j}$ would be scattered about a straight line, joining the points $(0,0)$ and $(0.5,1)$. On the other hand, non-randomness would produce non-random $a_{t}$ 's whose cumulative periodogram could show systematic deviations from the line. In particular, periodicities in the $a_{t}$ 's would tend to produce a series of neighbouring values of $I\left(f_{i}\right)$, which are large. These large ordinates would reinforce each other in $\mathrm{C}\left(\mathrm{f}_{\mathrm{i}}\right)$, and form a lump on the expected straight line.

Box and Jenkins (1976) have used approximate probability limits for cumulative periodogram using Kolmogorov-Smirnov test. The limits lines are drawn about the theoretical line which are such that, if the $\left\{a_{t}\right\}$ series were white noise, then the cumulative periodogram would deviate from the straight line, sufficiently to cross the limits, only with stated property. The limit lines are such that for a true random series, they would be crossed by a proportion of the time. They are drawn at distances $\pm \mathrm{K}_{\varepsilon} / \sqrt{\mathrm{q}}$ above and below the theoretical line, where $\mathrm{q}=$ $(n-2) / 2$ for $n$ even and $q=(n-1) / 2$ for $n$ odd where $\varepsilon$ is the 
level of significance. Approximate values of $K_{\varepsilon}$ are given in Table 1. With the availability of computer packages for diagnostic checking, the entire process reduces to the selection of the appropriate test depending on the parameters used.

Table 1. Coefficient for calculating approximate probability limits for cumulative periodogram test.

\begin{tabular}{|c|c|}
\hline$\varepsilon\{$ PRIVATE $\}$ & $\mathrm{K}_{\varepsilon}$ \\
\hline 0.01 & 1.63 \\
\hline 0.05 & 1.36 \\
\hline 0.10 & 1.22 \\
\hline 0.25 & 1.02 \\
\hline
\end{tabular}

Portmanteau statistic

1.6. 1. Portmanteau lack of fit test

Consider a discrete time series $\left\{w_{t}\right\}$ generated by a stationary autoregressive moving average model

$\varphi(B) w_{t}=\theta(B) a_{t}$, where $w_{t}=\nabla^{d} z_{t}$

Where $d$ is an integer or any real number and $\left\{z_{t}\right\}$ is any time series.
$\varphi(B)=1-\varphi_{1} B-$ $-\varphi_{p} B^{p}$
$\theta(B)=1-\theta_{1} B-\ldots \ldots-\theta_{q} B^{q}$

Here $w_{t}$ 's can in general represent the dth difference or some other suitable transformation of a non-stationary series $\left\{z_{\mathrm{t}}\right\}$. After a model of this form has been fitted to a series $w_{1}, w_{2}, \ldots, w_{n}$, it is useful to study the adequacy of the fit by examining the residuals $\hat{a}_{1}, \ldots ., \hat{a}_{1}$ and in particular their autocorrelations

$$
r_{k}(\widehat{a})=\frac{\sum_{t=k+1}^{n} \widehat{a}_{t} \widehat{a}_{t+k}}{\sum_{t=1}^{n} a_{t}^{2}}, \quad \mathrm{k}=1,2, \ldots \ldots
$$

A graphical analysis of these quantities combined with overfitting procedure given by Box and Jenkins (1976) usually prove to be most effective in detecting possible deficiencies in the model. Box and Pierce (1970) suggested that if the models were appropriate and parameters were known, the quantity

$\mathrm{Q}_{2}(\mathrm{r})=\mathrm{n}(\mathrm{n}+2) \sum_{k=1}^{m} \frac{r_{k}^{2}(a)}{(n-k)}$, where $\mathrm{r}_{\mathrm{k}}(\mathrm{a})=\frac{\sum_{t=k+1}^{n} a_{t} a_{t-k}}{\sum_{t=1}^{n} a_{t}{ }^{2}}$

Would for large $\mathrm{n}$ be distributed as $\mathrm{Xm}_{\mathrm{m}}{ }^{2}$ since the limiting distribution of $r=\left(r_{1}, \ldots \ldots, r_{m}\right)^{\prime}$ is multivariate normal with mean zero (Ljung \& Box, 1978).

$$
\begin{aligned}
& \operatorname{Var}\left(r_{k}(a)\right)=\frac{n-k}{n(n+2)} \\
& \operatorname{cov}\left(r_{k}(a), r(a)\right)=0 \quad(k \neq \emptyset)
\end{aligned}
$$

Using further approximation, $\operatorname{var}\left(r_{k}(a)\right)=1 / n$
Vol. 3 No. 9 (Sep 2010)

ISSN: 0974- 6846

Box and Pierce (1970) suggested that the distribution of

$$
\mathrm{Q}_{1}(\mathrm{r})=n \sum_{k=1}^{m} r_{k}^{2}(a)
$$

Could be approximated by that of $\mathrm{Xm}^{2}$. Further, they showed that when $\mathrm{p}+\mathrm{q}$ parameters of an appropriate model are estimated and the $r_{k}(\hat{a})$ 's replace the $r_{k}(a)$ 's, then

$$
\mathrm{Q}_{1}(\widehat{r})=n \sum_{k=1}^{m} r_{k}^{2}(\widehat{a})
$$

would for large $n$ be distributed as $\mathrm{X}_{\mathrm{m}-\mathrm{p}-\mathrm{q}}$

A modified test based on the criterion $Q_{2}(\widehat{r})=n(n+2)$

$\sum_{k=1}^{m} \frac{r_{k}^{2}(\widehat{a})}{(n-k)}$

Was recommended by Ljung and Box (1978). The modified test provides an improved approximation that should be adequate for most practical purposes. It is also noted by Davies et al. (1977) that the variance of $\mathrm{Q}_{2}(\widehat{r})$ exceeds that of the $\psi_{\mathrm{m} \text {-p } \mathrm{q}}^{2}$ distribution. The above criterion is further modified in this study to facilitate better approximation to the distribution and in turn to give better identification of the models.

The proposed $Q$ statistics are

$$
\begin{aligned}
& \mathrm{Q}_{3}(\mathrm{r})=n \sum_{k=1}^{m} \frac{n-k}{(n+k)} r_{k}^{2}(\widehat{a}) \text { and } \\
& \mathrm{Q}_{4}(\mathrm{r})=\mathrm{n}(\mathrm{n}+2) \sum_{k=1}^{m} \frac{r_{k}^{2}(\widehat{a})}{(n+k)}
\end{aligned}
$$

\subsubsection{Mean and variance of Portmanteau statistics}

The residual autocorrelation $r_{k}$ of the process is written in the form

$$
r_{k}(a)=\frac{\sum_{t=k+1}^{n} a_{t} a_{t-k}}{\sum_{t=1}^{n} a_{t}^{2}}, \quad \mathrm{k}=1,2, \ldots . .
$$

Then

$$
\begin{aligned}
& \mathrm{E}\left(\mathrm{r}_{\mathrm{k}}(\mathrm{a})\right)=\mathrm{E} \frac{\sum_{t=k+1}^{n} a_{t} a_{t-k}}{\sum_{t=1}^{n} a_{t}^{2}}=\frac{1}{n^{2} \sigma^{2}} E \sum_{t=k+1}^{n} a_{t} a_{t-k}=0 \\
& \operatorname{var}\left(\mathrm{r}_{\mathrm{k}}(\mathrm{a})\right)=\mathrm{E}\left(\mathrm{r}_{\mathrm{k}}^{2}(\mathrm{a})\right)=\frac{1}{n^{2} \sigma^{2}} E \sum_{t=k+1}^{n} a_{t} a_{t-k} \\
& =\frac{1}{n^{2} \sigma^{2}}\left(\mathrm{a}_{\mathrm{k}+1} \mathrm{a}_{1}+\mathrm{a}_{\mathrm{k}+1} \mathrm{a}_{2}+\ldots \ldots . .+\mathrm{a}_{\mathrm{n}} \mathrm{a}_{\mathrm{n}-\mathrm{k}}\right)^{2} \\
& =\frac{n-k}{n^{2}} \rightarrow \frac{1}{n} \text { for large } \mathrm{n} \\
& \text { It is found that (Davies et al., 1977), }
\end{aligned}
$$




$$
\begin{gathered}
\quad \operatorname{var}\left(\mathrm{r}_{\mathrm{k}}^{2}(\mathrm{a})\right)=\frac{6(3 n-5 k)+3(n-k)}{n(n+2)(n+4)(n+6)}-\frac{(n-k)^{2}}{n^{2}(n+2)^{2}} \\
\operatorname{cov}\left(\mathrm{r}_{\mathrm{k}}^{2}(\mathrm{a}), \mathrm{r}_{\mathrm{r}}^{2}(\mathrm{a})\right) \\
=\frac{(n-k)(n-l)+4(n-l)+8(n-k-l)}{n(n+2)(n+4)(n+6)}-\frac{(n-k)(n-l)}{n^{2}(n+2)^{2}}
\end{gathered}
$$

The $Q$ statistics and their variances of the existing ones and the proposed ones are given below

$$
\begin{aligned}
& \text { i) } \mathrm{Q}_{1}(\mathrm{r})=n \sum_{k=1}^{m} r_{k}^{2}(a)(\text { Box \& Pierce, 1970) } \\
& \operatorname{var}\left(Q_{1}(r)\right) \\
& \left.=n^{2} \sum_{k=1}^{m} \operatorname{var}\left(r_{k}^{2}(a)\right)+2 n^{2} \sum_{k=1}^{m-1} \sum_{l=k+1}^{m} \operatorname{cov}\left(r_{k}^{2}(a)\right), r_{l}^{2}(a)\right) \\
& \text { ii) } \mathrm{Q}_{2}(\mathrm{r})=\mathrm{n}(\mathrm{n}+2) \sum_{k=1}^{m} \frac{r_{k}^{2}(a)}{(n-k)} \quad \text { (Ljung \& Box 1978) } \\
& \operatorname{var}\left(\mathrm{Q}_{2}(\mathrm{r})\right)=\mathrm{n}^{2}(\mathrm{n}+2)^{2} \sum_{k=1}^{m} \frac{\left(r_{k}^{2}(a)\right)}{(n-k)} \\
& +2 n^{2}(\mathrm{n}+2)^{2} \sum_{k=1}^{m-1} \sum_{l=k+1}^{m} \frac{\operatorname{cov}\left(r_{k}^{2}(a), r_{1}^{2}(a)\right)}{(n-k)(n-l)}
\end{aligned}
$$$$
\text { iii) } \mathrm{Q}_{3}(\mathrm{r})=n \sum_{k=1}^{m} \frac{n-k}{(n+k)} r_{k}^{2}(a)
$$$$
\operatorname{var}\left(\mathrm{Q}_{3}(\mathrm{r})\right)=n^{2} \sum_{k=1}^{m} \frac{n-k^{2}}{(n+k)} \operatorname{var}\left(r_{k}^{2}(a)\right)
$$$$
+2 \mathrm{n}^{2} \sum_{k=1}^{m-1} \sum_{l=k+1}^{m} \frac{(n-k)(n-l)}{(n-k)(n+l)} \operatorname{cov}\left(r_{k}^{2}(a), r_{l}^{2}(a)\right)
$$$$
\text { iv) } \mathrm{Q}_{4}(\mathrm{r})=\mathrm{n}(\mathrm{n}+2) \sum_{k=1}^{m} \frac{r_{k}^{2}(a)}{(n+k)}
$$$$
\operatorname{var}\left(\mathrm{Q}_{4}(\mathrm{r})=\mathrm{n}^{2}(\mathrm{n}+2) \sum_{k=1}^{m} \frac{\operatorname{var}\left(r_{k}^{2}(a)\right)}{(n-k)^{2}}\right.
$$

Table 2. Mean and Variance of $Q_{i}(r), i=1,2,3,4$.

\begin{tabular}{|c|r|r|r|r|}
\hline $\begin{array}{c}\text { PPRIVATE \}n, } \\
m\end{array}$ & $\begin{array}{c}Q_{1}(r) \\
\text { Box \& Pierce }\end{array}$ & $\begin{array}{c}Q_{2}(r) \\
\text { Box \& Ljung }\end{array}$ & $Q_{3}(r)$ & $Q_{4}(r)$ \\
\hline$n=150, m=15$ & 28.240 & 43.022 & 16.408 & 23.605 \\
\hline$n=50, m=12$ & 22.435 & 31.974 & 14.217 & 19.516 \\
\hline$n=100, m=30$ & 63.1546 & 91.480 & 37.421 & 51.216 \\
\hline$n=100, m=25$ & 52.269 & 71.577 & 33.264 & 43.789 \\
\hline$n=100, m=20$ & 41.218 & 53.470 & 28.315 & 25.773 \\
\hline$n=100, m=50$ & 110.608 & 147.672 & 71.090 & 91.257 \\
\hline$n=100, m=45$ & 48.859 & 128.380 & 65.978 & 82.994 \\
\hline$n=100, m=30$ & 63.842 & 76.425 & 48.051 & 56.705 \\
\hline$n=100, m=75$ & 169.113 & 223.871 & 109.049 & 138.810 \\
\hline$n=100, m=50$ & 109.294 & 132.075 & 80.285 & 95.329 \\
\hline$n=400, m=100$ & 227.662 & 300.099 & 147.043 & 186.386 \\
\hline$n=400, m=75$ & 167.085 & 205.634 & 118.854 & 143.069 \\
\hline$n=400, m=50$ & 107.767 & 124.056 & 85.053 & 96.937 \\
\hline
\end{tabular}

$Q_{i}(r), i=1,2,3,4$ are asymptotically distributed as $x_{m}{ }^{2}$ and have expectation $m$ and variance $2 m$. Variance of $Q_{i}(r)$, i $=1,2,3,4$ are tabulated in Table 2 for comparative study for different values of $n$ and $m$.

Diagnostic checks have become a standard tool for assessing the adequacy of a forecasting system, since ARIMA modeling techniques (Box \& Jenkins, 1976) became popular. Box and Pierce (1970) gave an overall test of fit which is based on the residual autocorrelation. Davies et al. (1977) suggested that there are cases in which low values of the portmanteau statistic are often found and in such cases, the true significance levels are likely to be much lower than predicted by asymptotic theory. Ljung and Box (1978) have shown that a substantially improved approximation results from a simple modification of Box and Pierce (1970) portmanteau test for transfer function-noise models. A simple statistic to cheek model adequacy in time series is also suggested by Abraham and Vijayan (1988). Most of the checks are available only for normal and second order stationary models. Smith (1985) has given various diagnostic checks that can be performed simply on nonnormal and non-standard models. Anders Milhqj (1981) proposed a goodness of fit and flat statistic for time series models in which the asymptotic power of the tests found are compared to the empirical power of the portmanteau test.

Analysis of Chennai city rainfall data

Models are identified for the different time series using the procedure given by Box and Jenkins (1976). The best model is chosen with help of modified portmanteau lack of fit test.

\section{Model identification}

The general behaviour of the estimated autocorrelation and partial autocorrelation function of the appropriate differenced series will give the clues about the choice of the orders of $p, q, P$ and $Q$ for the autoregressive and moving average operators for non-stationary seasonal model to identify the multiplicative ARIMA process (Box \& Jenkins, 1976). The rainfall data of the Chennai city for the period 1981 to 1994 is taken for analysis. The given series $z_{t}$ is transformed by adding a constant $C$. The transformation of the original asymmetric periodic time series $z_{t}$ into a normally distributed time series $Z_{t}$ is done by taking logarithms of $z_{t}+C$. The value of $C$ is usually assumed to be lower bound of the series or it is chosen arbitrarily.

The autocorrelation (Table 3) and partial autocorrelation of the transformed series $z_{t}$ do not render any help for model identification except that the given time series is seasonal with period 12 . The seasonality in the series is removed by taking twelfth difference. The 
Table 3. Autocorrelation of the series $z_{t}$ and $\left(1-B^{12}\right) z_{t}$

\begin{tabular}{|c|c|c|c|}
\hline lag k & Autocorrelation of $\mathrm{Z}_{\mathrm{t}}$ & lag $\mathrm{k}$ & Autocorrelation of $\left(1-\mathrm{B}^{12}\right) \mathrm{Z}_{\mathrm{t}}$ \\
\hline 1 & & & \\
\hline 2 & 0.500 & 1 & -0.230 \\
\hline 3 & 0.286 & 2 & 0.073 \\
\hline 4 & -0.070 & 3 & -0.082 \\
\hline 5 & -0.286 & 4 & 0.067 \\
\hline 6 & -0.483 & 5 & -0.062 \\
\hline 7 & -0.553 & 6 & -0.068 \\
\hline 8 & -0.448 & 7 & 0.143 \\
\hline 9 & -0.354 & 8 & -0.090 \\
\hline 10 & -0.058 & 9 & 0.066 \\
\hline 11 & -0.220 & 10 & 0.031 \\
\hline 12 & -0.537 & 11 & 0.080 \\
\hline 13 & -0.631 & 12 & -0.499 \\
\hline 14 & -0.526 & 13 & 0.118 \\
\hline 15 & -0.255 & 14 & 0.034 \\
\hline 16 & -0.046 & 15 & -0.049 \\
\hline 17 & -0.248 & 16 & 0.078 \\
\hline 18 & -0.456 & 17 & -0.058 \\
\hline 19 & -0.472 & 18 & 0.110 \\
\hline 20 & -0.457 & 19 & -0.119 \\
\hline 21 & -0.325 & 20 & 0.034 \\
\hline 22 & -0.059 & 21 & 0.015 \\
\hline 23 & -0.153 & 22 & -0.082 \\
\hline 24 & 0.509 & 23 & 0.123 \\
\hline 25 & 0.576 & 24 & 0.063 \\
\hline 26 & 0.467 & 25 & -0.062 \\
\hline 27 & 0.202 & 26 & -0.136 \\
\hline 28 & -0.011 & 27 & 0.129 \\
\hline 29 & -0.257 & 28 & -0.077 \\
\hline 30 & -0.499 & 29 & 0.017 \\
\hline 245 & 30 & -0.080 \\
\hline
\end{tabular}

autocorrelation of the differenced time series (Table 3 ) indicates that it has large autocorrelation only at lag 12 After trying many values for $\mathrm{C}$ the appropriate value of $\mathrm{C}$ is found to be 15.0 .

In this case the additive constant is necessitated because Chennai city did not experience any amount of rain in some months. As with the non-seasonal model by equating observed autocorrelation to their expected values, approximate values can be obtained for the parameter $\Theta(\theta$ $=0$ ). On substituting the estimation $r_{12}$ in the place of $\rho_{12}$ in the equation:

$\rho_{12}=\frac{-\Theta}{1+\Theta^{2}}$

We obtain the initial estimate for $\Theta$ as $\Theta=0.8013$. The ARIMA model for the rainfall data can be written in the form $\nabla_{12} z_{t}=\left(1-\Theta B^{12}\right) a_{t}$

The value of $\Theta$ can be estimated by using either least squares estimate method or estimation by numerical derivations.

The above model can be written in the form.

$a_{t, 0}=\left(\Theta-\Theta_{0}\right) x_{2, t}+a_{t}$
Wherex $x_{2, t}=\frac{-\partial a_{t}}{\partial \Theta}$ and $\Theta_{0}$ is the assumed value of $\mathrm{a}_{\mathrm{t}, 0}=\left[\mathrm{a}_{\mathrm{t}} / \Theta_{0}\right]$

The derivatives are most easily computed numerically by

$\mathrm{x}_{2, \mathrm{t}}=\frac{\left[a_{t}\right]-\left[a_{t, 0}\right]}{\delta}, \delta>0$ which is very small.

To obtain the first adjustment for $\Theta$, we compute

$$
\Theta-\Theta_{\circ}=\frac{\sum_{t=0}^{n}\left[a_{t, 0}\right]}{\sum_{t=0}^{n} x_{t}^{2}}, \mathrm{n}=\mathrm{N}-\mathrm{sD}=156
$$

The value of $\Theta$ is estimated as $\widehat{\Theta}=0.9265$ (Table 4). The model is written in the form

$$
\nabla^{12} z_{t}=\left(1-\widehat{\Theta} B^{12}\right) a_{t} \quad \text { where } \widehat{\Theta}=0.9265 \text {. }
$$

Table 4. Iterative estimation of $\Theta$ for the rainfall data.

\begin{tabular}{|c|c|}
\hline \{PRIVATE \}lteration & $\Theta$ \\
\hline 1 & 0.9291 \\
\hline 2 & 0.9268 \\
\hline 3 & 0.9265 \\
\hline 4 & 0.9265 \\
\hline 5 & 0.9265 \\
\hline 6 & 0.9265 \\
\hline 7 & 0.9265 \\
\hline 8 & 0.9265 \\
\hline 9 & 0.9265 \\
\hline 10 & 0.9265 \\
\hline
\end{tabular}

\section{Portmanteau lack of fit test}

After identifying the model, it is customary to validate the model before forecasting the data. The proposed portmanteau criteria and the existing tests are applied and the model is then identified.

In the case of ARIMA model (Box \& Jenkins, 1976), We refer $Q_{3}(r)=37.0563$ to a $X^{2}$ table with 47 degrees of freedom. The $90 \%$ and $75 \%$ for $x^{2}$ with 47 degrees of freedom are 30.7 and 37.3 (Table 5). It shows that there is no basis for questioning this model and that it can be used for further analysis.

\begin{tabular}{|c|c|c|c|}
\hline $\begin{array}{c}\text { PPRIVAT } \\
E \text { \} } Q_{i}(r) \\
i=1,2,3,4\end{array}$ & $\begin{array}{l}\text { Residual } \\
\text { square sum }\end{array}$ & \multicolumn{2}{|c|}{$\begin{array}{l}\mathrm{x}^{2} \text { distribution with } 47 \text { degrees of } \\
\text { freedom }\end{array}$} \\
\hline \multirow{2}{*}{$\mathrm{Q}_{1}(1)$} & \multirow{2}{*}{47.7441} & $50 \%$ & $25 \%$ \\
\hline & & 45.6 & 55.3 \\
\hline \multirow{2}{*}{$Q_{2}(r)$} & \multirow{2}{*}{56.4925} & $25 \%$ & $10 \%$ \\
\hline & & 55.3 & 65.1 \\
\hline \multirow{2}{*}{$Q_{3}(r)$} & \multirow{2}{*}{37.0563} & $90 \%$ & $75 \%$ \\
\hline & & 30.7 & 37.3 \\
\hline \multirow{2}{*}{$Q_{4}(r)$} & \multirow{2}{*}{42.9438} & $75 \%$ & $50 \%$ \\
\hline & & 37.3 & 45.6 \\
\hline
\end{tabular}

Table 5. Comparison of sum of the squared residuals. 


\section{Forecasting}

Forecasting can be done from the difference equation itself. The ARIMA model for the rainfall data written in the form at lead time / where we are currently standing at time $t$ is given by

$$
\begin{aligned}
& \nabla_{12} z_{t+1}=\left(1-\widehat{\Theta} B^{12}\right) a_{t+1} \text {, where } \widehat{\Theta}=0.9265 \text {. } \\
& z_{t+1}-z_{t+12}=a_{t+1}-\widehat{\Theta} a_{t+1-12} \\
& z_{t+1}=z_{t+\ell-12}-\widehat{\Theta} a_{t+\ell-12}+a_{t+1}
\end{aligned}
$$

The minimum mean square error forecast at lead time $\ell$ and at origin $\mathrm{t}$ is given by

$$
\widehat{\mathrm{z}}_{\mathrm{t}=}\left[\mathrm{z}_{\mathrm{t}++12}\right]-\widehat{\Theta}\left[\mathrm{a}_{\mathrm{t}++12}\right)+\left[\mathrm{a}_{\mathrm{t}+}\right] .
$$

\section{References}

1. Abraham B and Vijayan K (1988) A statistic to check model adequacy in time series. Comm. Statist. Theory Methods. 17(12), 4271-4278.

2. Anders Milhqj (1981) A test of fit in time series models. Biometrika, 68, 1, 177-87.

3. Anderson RL (1941) Distribution of the time serial correlation coefficients. Ann. Math. Stat. 8(1), 1-13.

4. Bartlett MS (1946) On the theoretical specification of sampling properties of autocorrelated time series. $J$. Royal Stat. Soc. Ser B. 8, 27-41.

5. Box GEP and Jenkins GM (1976) Time series analysis: forecasting and control. Revised edition, San Francisco: Holden-day.

6. Box GEP and Pierce DA (1970) Distribution of residual autocorrelation in autoregressive integrated moving average models. J. Amer. Stat. Assoc. 64, 1509-1526.
Vol. 3 No. 9 (Sep 2010)

ISSN: 0974- 6846

7. Box GEP, Jenkins GM and Reinsel GC (1994) Time series analysis: forecasting and control, $3^{\text {rd }}$ edition, Prentice Hall, New Jersey.

8. Brockwell BJ and Davis RA (1991) Time series: theory and methods, $2^{\text {nd }}$ edition, Springer-Verlag, New York.

9. Davies N, Triggs CM and Newbold P (1977) Significance levels of the Box-Pierce Portmanteau statistic in finite samples. Biometrika. 64(3), 517-522.

10.Godfrey LG (1979) Testing the adequacy of a time series model. Biometrika. 66, 67-72.

11. Hokstad P (1983) A method for diagnostic checking of time series models. J. Time Ser. Anal. 4(3), 177-183.

12.Jenkins GM and Watts DG (1968) On the theoretical specification of sampling properties of autocorrelated time series. J. Royal Stat. Soc. B8, 27.

13. Jenkins GM and Watts DG (1969) Spectral analysis and its applications, Holden-day series in time series analysis, San Francisco, California.

14.Ljung GM (1986) Diagnostic testing of univariate time series models. Biometrika. 73(3), 725-730.

15.Ljung GM and Box GEP (1978) On a measure of lack of fit in time series models. Biometrika. 65, 297-303.

16. McLeod Al (1994) Diagnostic checking of periodic auto regression models with application. J. Time Ser. Anal. 15(2), 221-233.

17.McLeod Al and Li WK (1983) Diagnostic checking ARMA time series models using squared-residual autocorrelation. J. Time Ser. Anal. 4(4), 269-273.

18.Smith JQ (1985) Diagnostic checks of non-standard time series models. J. Forecasting. 4, 283-291. 